\title{
Neue Studien zur Hypertonie
}

\author{
Ina Zivanovic, Sebastian Ewen, Michael Böhm, Felix Mahfoud
}

\begin{tabular}{|c|c|}
\hline \multicolumn{2}{|l|}{ Übersicht } \\
\hline Einleitung & 237 \\
\hline Neue Blutdruckzielwerte? & 237 \\
\hline Therapie-Update etablierter & \\
\hline Antihyper- & \\
\hline tensiva & 239 \\
\hline $\begin{array}{l}\text { Therapie-Innovationen - } \\
\text { die Angiotensinrezeptor- }\end{array}$ & \\
\hline Neprilysin-Inhibitoren & 240 \\
\hline $\begin{array}{l}\text { Behandlungsoptionen bei } \\
\text { therapieresistenter Hypertonie }\end{array}$ & 241 \\
\hline
\end{tabular}

\section{Einleitung}

Die arterielle Hypertonie gehört weltweit zu den häufigsten chronischen Erkrankungen und ist ein Hauptrisikofaktor für kardiovaskuläre Morbidität und Sterblichkeit [1]. Die nachfolgende Übersicht diskutiert neue Studienergebnisse aus der Hypertonieforschung der letzten beiden Jahre. Zu Beginn der Arbeit werden neue Erkenntnisse zu Blutdruckzielwerten vorgestellt, nachfolgend aktuelle Empfehlungen zu Medikamentenregimen sowie neue pharmakologische Entwicklungen erläutert. Abschließend wird auf aktuelle Erkenntnisse medikamentöser und nicht medikamentöser Therapieoptionen bei therapieresistenter Hypertonie eingegangen.

\section{Neue Blutdruckzielwerte?}

\section{Leitlinie}

Die gemeinsame Leitlinie der European Society of Cardiology (ESC) und der European Society of Hypertension (ESH) empfiehlt allgemein einen Zielblutdruck $<140 / 90 \mathrm{mmHg}$ und einen ambulanten 24-StundenZielblutdruck < 130/80 mmHg (Tab. 1) [2]. Außerdem empfiehlt die Leitlinie:
- Für ältere Patienten (> 80 Jahre) gilt ein systolischer Zielblutdruck von 140-150 mmHg bei initialen Blutdruckwerten $>150 \mathrm{mmHg}$.

- Bei Patienten mit Diabetes mellitus sollte der diastolische Zielblutdruck bei $80-85 \mathrm{mmHg}$ liegen.

- Bei Patienten mit Proteinurie gilt ein systolischer Zielblutdruck von $<130 \mathrm{mmHg}$.

Die Diskussion der optimalen Zielblutdruckwerte ist aufgrund der Ende September 2015 vorzeitig beendeten US-amerikanischen SPRINT-Studie (SPRINT = Systolic blood PRessure Intervention Trial) erneut entfacht [3]. Grundsätzlich werden 2 Therapieprinzipien diskutiert [2]:

- die Maxime ,je niedriger, desto besser“

- das Prinzip der „J-Kurve“

\section{SPRINT-Studie}

Ziel der SPRINT-Studie war es festzustellen, ob ein systolischer Blutdruck (SBP) unter $120 \mathrm{mmHg}$ im Vergleich zu einem SBP unter $140 \mathrm{mmHg}$ mit einer Reduktion kardiovaskulärer Ereignisse einhergeht [3]:

- Die multizentrische, randomisierte Studie umfasste 9361 Patienten im Alter von > 50 Jahren, einem $\mathrm{SBP} \geq 130 \mathrm{mmHg}$ und zusätzlich mindestens einem der folgenden Risikofaktoren: Herz-KreislaufErkrankung, chronische Niereninsuffizienz (glomeruläre Filtrationsrate [GFR] zwischen 20 und 


\section{Tabelle 1}

Leitlinien zu empfohlenen Blutdruckzielwerten bei verschiedenen Patientengruppen [2].

\begin{tabular}{|c|c|c|}
\hline Patientengruppe & SBP-Zielwert & DBP-Zielwert \\
\hline alle Patienten, unabhängig vom Risiko & $<140 \mathrm{mmHg}$ & $<90 \mathrm{mmHg}$ \\
\hline Patienten > 80 Jahre & $140-150 \mathrm{mmHg}$ & \\
\hline $\begin{array}{l}\text { Patienten mit Nephropathie und Proteinurie } \\
\geq 300 \mathrm{mg} / \mathrm{d}\end{array}$ & $<130 \mathrm{mmHg}$ & \\
\hline Patienten mit Diabetes mellitus & $<140 \mathrm{mmHg}$ & $80-85 \mathrm{mmHg}$ \\
\hline
\end{tabular}

$59 \mathrm{ml} / \mathrm{min} / 1,73 \mathrm{~m}^{2}$ ), 10-Jahres-Framingham-RisikoScore $\geq 15 \%$ oder Alter $>75$ Jahren.

- Von der Untersuchung ausgeschlossen wurden Patienten mit symptomatischer Herzinsuffizienz oder linksventrikulärer Pumpfunktion unter 35\%, Zustand nach Schlaganfall, Diabetes mellitus, polyzystischer Nierenerkrankung, Proteinurie $\geq 1 \mathrm{~g} / \mathrm{d}$ sowie einer GFR unter $20 \mathrm{ml} / \mathrm{min} / 1,73 \mathrm{~m}^{2}$.

- Die Studie wurde am 11.September 2015 ca. 2 Jahre vor geplantem Studienende von der Studienleitung abgebrochen, da sich in einer Zwischenauswertung eine signifikante Überlegenheit des niedrigeren Zielblutdrucks zeigte [4]: In der intensivierten Behandlungsgruppe gab es $27 \%$ weniger Todesfälle und $25 \%$ weniger kardiovaskuläre Ereignisse als in der Gruppe mit einem Zielblutdruck unter $140 \mathrm{mmHg}$. Die Ergebnisse der SPRINT-Studie deuten darauf hin, dass niedrigere SBP-Zielwerte (<120 mmHg) für Patienten mit erhöhtem kardiovaskulärem Risiko anzustreben sind. Eine kürzlich veröffentliche Metaanalyse, die ca. 50000 Patienten einschloss, bestätigte eine kardiovaskuläre Risikoreduktion für niedrigere Blutdruckwerte [5].

Kritisch anzumerken ist jedoch, dass 2 große Patientengruppen mit ebenfalls erhöhtem kardiovaskulärem Risiko in der SPRINT-Studie per Protokoll ausgeschlossen worden wurden [3]:

- Patienten mit Diabetes mellitus

- Patienten nach einem Schlaganfall
Interessanterweise zeigte die ACCORD-Studie bei Patienten mit Diabetes, dass eine SBP-Reduktion unter $120 \mathrm{mmHg}$ keinen positiven Effekt auf kardiovaskuläre Ereignisse hatte [6]. Die bislang durchgeführten Studien zu Blutdruckzielwerten bei Patienten mit stattgehabten Schlaganfall konnten ebenfalls bislang keinen klaren Zielwert ermitteln [7,8]. Bei der Bewertung der Ergebnisse der SPRINT-Studie sollte ebenfalls berücksichtigt werden, dass:

- 3 konsekutive Blutdruckmessungen mit einem automatischen, programmierten Messgerät durchgeführt wurden, was einen potenziellen „Weißkittel-Effekt“ nahezu ausschließt [2]

- im Gegensatz zur gegenwärtigen Praxis in Europa größtenteils Chlorthalidon anstatt Hydrochlorothiazid (HCT) als Diuretikum eingesetzt wurde [3].

Die Therapieempfehlungen basierten im Wesentlichen auf dem Behandlungsalgorithmus der aktuellen Europäischen Leitlinien und einem studienspezifischen empfohlenen Algorithmus, wurden jedoch in ihrer Ausführung den Prüfärzten überlassen. Zusammenfassend stellt sich die Frage, ob die Empfehlungen der Leitlinie mit einem allgemeingültigen Zielwert ausreichend sind, oder ob die Anamnese bzw. Komorbiditäten in einen für jeden Patienten individuell festgelegten Zielwert zukünftig noch stärker gewichtet werden sollten.

SPRINT: Bei hypertonen Hochrisikopatienten reduziert ein systolischer Zielwert unter $120 \mathrm{mmHg}$ im Vergleich zu einem Zielwert unter $140 \mathrm{mmHg}$ kardiovaskuläre Ereignisse um $25 \%$ und die Sterblichkeit um ein $27 \%$.

\section{Therapie-Update etablierter Antihypertensiva}

Die aktuelle Leitlinie der ESC/ESH empfiehlt einen Therapiealgorithmus, der maßgeblich auf einer Behandlung mit ACE-Hemmer/Angiotensinrezeptorblocker, Kalziumkanalblocker und Thiaziddiuretika basiert (Abb. 1) [2]. Betablocker kommen bei kardialer Komorbidität zum Einsatz.

Die empfohlene Primärtherapie für Bluthochdruckpatienten mit Diabetes ist ein ACE-Hemmer, bei Unverträglichkeit sollte ein Angiotensinrezeptorblocker eingesetzt werden [2]. Sollte der Blutdruck unter dieser Medikation noch oberhalb des von den Leitlinien empfohlenen Zielwerts liegen, ist im 
nächsten Schritt ein Kalziumantagonist vom Dihydropyridin-Typ zu ergänzen.

\section{Neubewertung des Einsatzes von Diuretika bei Patienten mit gestörter Glukose- toleranz}

\section{Wirkungsmechanismus der Thiaziddiuretika}

Diuretika, vor allem Thiaziddiuretika, können eine zusätzliche Störung der Glukosetoleranz verursachen, sodass ihre Anwendung bei Patienten mit gestörter Glukosetoleranz kontrovers diskutiert wird. Bei einer Langzzeittherapie mit einem Thiaziddiuretikum allein oder einer Kombination ist die Inzidenz neuer Hyperglykämien bzw. eines Diabetes mellitus Typ 2 erhöht. Dies ist mit großer Wahrscheinlichkeit dadurch bedingt, dass die Serumkaliumkonzentration sinkt und damit die Insulinsekretion gehemmt wird.

\section{PATHWAY-3-Studie}

Vor dem Hintergrund der Auswahl eines geeigneten Diuretikums bei Patienten mit gestörter Glukosetoleranz wurde die PATHWAY-3-Studie $($ PATHWAY $=$ Prevention And Treatment of Hypertension With Algorithm based therapY) konzipiert, deren Ergebnisse auf dem ESC-Kongress 2015 in London vorgestellt wurden [9]. Die Studie untersuchte die Effekte von HCT, Amilorid und deren Kombination bei Patienten mit Hypertonie in Bezug auf die Glukosetoleranz und Blutdruckreduktion.

- Die Studie war dreiarmig, randomisiert und doppelblind angelegt und untersuchte 441 Patienten mit einem unkontrollierten Blutdruck (Praxis-SBP $\geq 140 \mathrm{mmHg}$ und häuslicher $\mathrm{SBP} \geq 130 \mathrm{mmHg}$ ), die mindestens einen charakteristischen Faktor des metabolischen Syndroms aufwiesen [9]. Der Studienzeitraum betrug 24 Wochen, eine bereits initiierte blutdrucksenkende Therapie spielte keine Rolle.

- Die Patienten wurden nach Einnahme eines Placebos für 4 Wochen in eine der 3 Behandlungsgruppen randomisiert und erhielten als Studienmedikation entweder Amilorid (10 mg), HCT ( $25 \mathrm{mg}$ ) oder eine Kombination beider Präparate in halber Dosis. Nach 12 Wochen wurde die Dosis verdoppelt.

- Patienten in der HCT-Behandlungsgruppe wiesen signifikant höhere Blutzuckerwerte in oralen Glukosetoleranztests (OGTT) auf als Patienten in der Amilorid- oder in der Amilorid-HCT-Kombinationsgruppe [10]. Der Unterschied zum Untersuchungsbeginn zwischen Amilorid und HCT nach 12 bzw. 24 Wochen lag bei $-0,55 \mathrm{mmol} / \mathrm{l}$. Das Kombinationspräparat

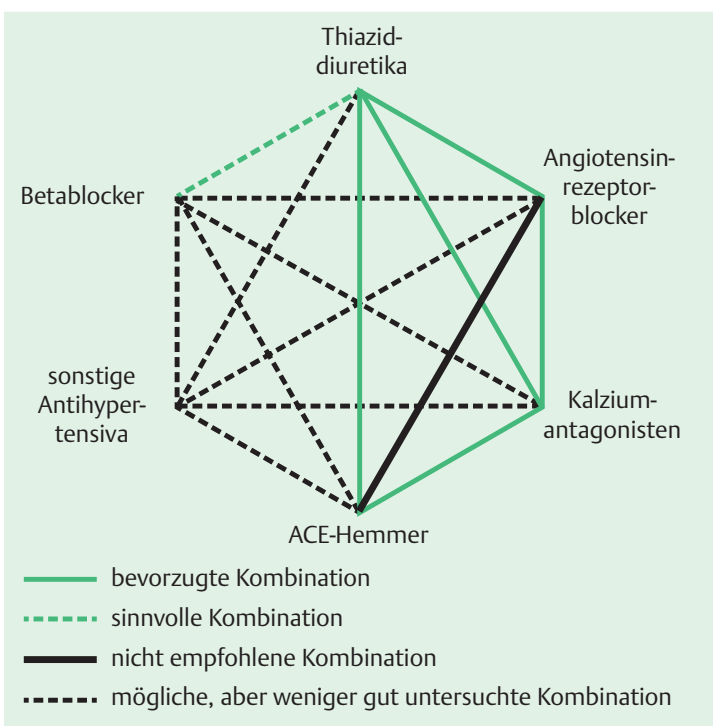

Abb. 1 Therapiealgorithmus der ESC/ ESH-Leitlinie Hypertonie (nach [2]).

unterschied sich von HCT nach 12 und 24 Wochen im OGTT-Ergebnis um - 0,42 mmol/l

- Beide Monotherapien reduzierten den häuslichen SBP vergleichbar gut (HCT - 12,2 mmHg, Amilorid $12,9 \mathrm{mmHg})$, die Reduktion unter der Kombinationstherapie war jedoch signifikant größer $(-15,6 \mathrm{mmHg})$.

- Der Plasmakaliumspiegel erhöhte sich unter Amilorid-Therapie, verringerte sich unter Medikation mit HCT und blieb unter Medikation mit dem Kombinationspräparat unverändert.

Zusammenfassend erzielte also die Kombination von HCT und Amilorid die größte Blutdrucksenkung im Vergleich zu einer Therapie mit den jeweiligen Einzelsubstanzen ohne signifikante Veränderungen des Kaliumspiegels und der Glukosekonzentration [10].

Die Kombination von Amilorid und HCT ist eine sichere und effektive Therapieoption für hypertone Patienten mit Glukoseintoleranz.

\section{ARB-induzierte Änderungen von Biomarkern - ATTEMPT-CVD-Studie}

Eine langjährige Hypertonie mit linksventrikulärer Hypertrophie ist mit einem Anstieg kardialer Biomarker assoziiert [2]. Eine erhöhte Albuminausscheidung im Urin ist ein früher Marker einer Nierenschädigung. Die ATTEMPT-CVD-Studie untersuchte den Effekt auf kardiovaskuläre Biomarker und auf kardiovaskuläre Ereignisse, den eine Bluthochdrucktherapie mit dem Angiotensinrezeptorblocker (ARB) Telmisartan im Ver- 
gleich zu einer Standardtherapie ohne Angiotensinrezeptorblocker hat [11].

- Die multizentrische, offene Studie randomisierte insgesamt 1228 Patienten (615 Patienten in einer Therapie mit Telmisartan, 613 Patienten in einer Standard-Bluthochdrucktherapie ohne Angiotensinrezeptorblocker) mit Bluthochdruck (Praxis-SBP $>140 \mathrm{mmHg}$ ) im Alter zwischen 40 und 80 Jahren, die einen oder mehrere kardiovaskuläre Risikofaktoren aufwiesen.

- Die Medikation wurde intensiviert bzw. erweitert, bis die von den Leitlinien vorgegebenen Zielblutdruckwerte $(\mathrm{SBP}<140 \mathrm{mmHg}, \mathrm{DBP}<90 \mathrm{mmHg}$ ) erreicht wurden.

- Die Telmisartan-Gruppe verzeichnete - unabhängig vom blutdrucksenkenden Effekt - eine geringfügige Erhöhung der BNP-Konzentration (BNP=B-Typ natriuretisches Peptid) und eine stärkere Senkung des Albumin/Kreatinin-Quotienten im Urin. Ein signifikanter Unterschied zwischen den Gruppen in Bezug auf kardiovaskuläre Ereignisse konnte nicht festgestellt werden.

Die ATTEMPT-CVD-Studie dokumentierte somit die positiven Effekte einer AngiotensinrezeptorblockerTherapie auf Plasma-BNP-Konzentration und den UrinAlbumin/Kreatinin-Quotienten, zeigte jedoch keinen zusätzlichen protektiven Einfluss auf kardiovaskuläre Ereignisse im Vergleich zu einer Therapie mit ACEHemmern, Kalziumantagonisten oder Diuretika.

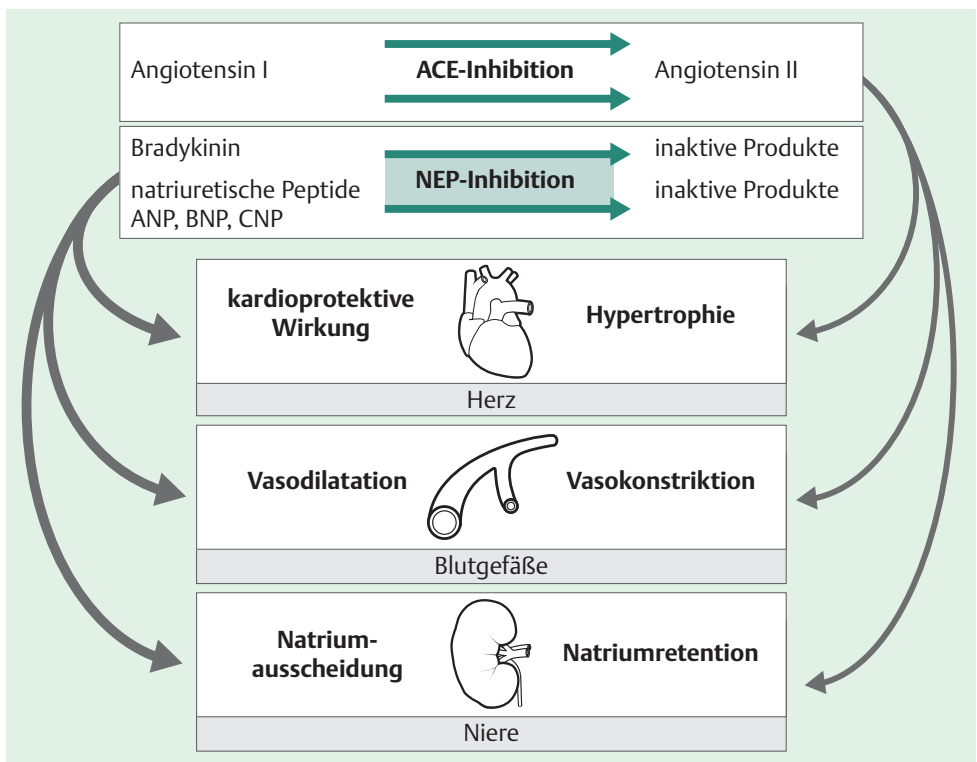

Abb. 2 Therapiekonzept des ARNI; $A C E=$ Angiotensin konvertierendes Enzym, $\mathrm{ANP}=$ atriales natriuretisches Peptid, $\mathrm{ARNI}=$ Angiotensinrezeptor-Neprilysin-Inhibitor, $\mathrm{BNP}=\mathrm{B}$-Typ natriuretisches Peptid, CNP $=\mathrm{C}$-Typ natriuretisches Peptid, NEP $=$ Neprilysin.

\section{Therapie-Innovationen - die Angiotensinrezeptor- Neprilysin-Inhibitoren}

\section{Wirkmechanismen}

Die neuroendokrine Aktivierung des Renin-Angiotensin-Systems (RAS) bei arterieller Hypertonie ist gut charakterisiert und Ansatzpunkt einer Reihe von antihypertensiven Substanzen [2]. Gegenregulatorische Systeme sind die sog. natriuretischen Peptide (atriales natriuretisches Peptid [ANP], B-Typ natriuretisches Peptid [BNP] und C-Typ natriuretisches Peptid [CNP]), die neben einer klinisch relevanten Vasodilatation und vermehrten Natriumausscheidung auch eine Kardioprotektion an isolierten Kardiomyozyten bewirken [12]. Die pathophysiologischen Wirkmechanismen sind in Abb. 2 zusammengefasst.

\section{Hybridmolekül LCZ696}

Die Kopplung des Angiotensinrezeptorblockers Valsartan mit dem Neprilysininhibitor Sacubitril führt über eine synergistische Wirkung mit Hemmung des RAS auch zu einem verminderten Abbau der natriuretischen Peptide. Das Hybridmolekül LCZ696 (Angiotensinrezeptorblocker und Neprilysininhibitor Sacubitril; „ARNI“) wird in der Darmmukosa in beide Komponenten gespalten, was zu einer stabilen Stöchiometrie beider Substanzen im Plasma führt [13]. Bei Patienten mit leicht bis moderat erhöhtem Blutdruck senkte eine Therapie mit LCZ696 den Blutdruck effektiver als Valsartan in der Monotherapie [13]. Damit stellte sich die Frage, ob LCZ696 auch einen besseren Schutz vor kardiovaskulären Komplikationen bei Patienten mit isoliert systolischer Hypertonie und arterieller Steifheit bieten könnte.

\section{PARAMETER-Studie}

Ziel der PARAMETER-Studie (PARAMETER = Prospective Comparison of ARNI with ARB Measuring Arterial Stiffness in the Elderly) war es, die Wirkung von LCZ696 auf die zentrale Aortenhämodynamik und arterielle Steifheit, im Vergleich zu Olmesartan, zu untersuchen [19]:

- Die multizentrische, randomisierte, doppelblinde Studie untersuchte 454 Patienten im Alter über 60 Jahre mit einem SBP $\geq 150 \mathrm{mmHg}$ und einem Pulsdruck > $60 \mathrm{mmHg}$ über 52 Wochen. 


\section{Hintergrundinformation}

\section{Misserfolge und Meilensteine der Neprilysininhibitior-Forschung}

- Eine isolierte Neprilysininhibition mit Candoxatril führte bei Patienten mit essenzieller Hypertonie nicht zu einer signifikanten Blutdrucksenkung [14].

- Eine gleichzeitige Hemmung des Angiotensin konvertierenden Enzyms und des Neprilysins mit Omapatrilat zeigte bei Patienten mit Bluthochdruck (OVERTURE-Studie) [15] und bei Patienten mit chronischer Herzinsuffizienz (OCTAVE-
Studie) [16] Vorteile verglichen mit Enalapril, verursachte jedoch schwere Angioödeme.

- Bei Patienten mit chronischer Herzinsuffizienz und einer reduzierten Pumpfunktion war Valsartan/Sacubitril in der Reduktion von Todesfällen und Hospitalisierungen einer Therapie mit Enalapril überlegen (PARADIGM-HF-Studie) [17].
- Verglichen mit Valsartan zeigte Valsartan/Sacubitril bei Patienten mit Hypertonie eine größere und komplementäre Blutdrucksenkung [13] und bei Patienten mit Herzinsuffizienz und erhaltener Pumpfunktion eine positive Änderung des NT-proBNP, der NYHA-Klasse, der GFR und der Dimension des rechten Vorhofs (PARAMOUNT-Studie) [18].
- Die Interventionsgruppe wurde auf LCZ696 $400 \mathrm{mg}$ und die Kontrollgruppe auf Olmesartan $40 \mathrm{mg}$ täglich titriert. Um einen Blutdruck < 140/90 mmHg zu erreichen, konnten im Anschluss an die ersten 12 Wochen Amlodipin und HCT zur Medikation ergänzt werden.

- Eine 12-wöchige Therapie mit LCZ696 führte im Vergleich zur Olmesartan-Monotherapie zu einer signifikant größeren Reduktion des Aorten-SBP $(-3,7 \mathrm{mmHg})$, des zentralen Pulsdrucks $(-2,4 \mathrm{mmHg})$, des brachialen SBP $(-3,8 \mathrm{mmHg})$, des brachialen Pulsdrucks $(-2,8 \mathrm{mmHg})$ und des zentralen $(-3,35 \mathrm{mmHg})$ und brachialen $(-4,10 \mathrm{mmHg})$ 24-Stunden-Blutdrucks, besonders in der nächtlichen Periode [20].

- Beide Medikamente senkten die NT-proBNP-Konzentration - LCZ696 signifikant mehr (34\%) als Olmesartan (20\%).

- Die LCZ696-Gruppe benötigte außerdem eine geringere Anzahl an zusätzlicher Begleitmedikation, um den prädefinierten Zielblutdruckwert zu erreichen. Das Nebenwirkungsprofil beider Testsubstanzen war vergleichbar.

LCZ696 reduzierte bei Hochrisikopatienten mit isoliert systolischer Hypertonie und einem erhöhten Pulsdruck den zentralen Blutdruck sowie den zentralen Pulsdruck effektiver als eine Monotherapie mit einem Angiotensinrezeptorblocker.

\section{Behandlungsoptionen bei the- rapieresistenter Hypertonie}

Etwa 5-15\% aller Patienten mit Bluthochdruck weisen eine therapieresistente arterielle Hypertonie auf, die mit einem deutlich erhöhten kardiovaskulären Risiko assoziiert ist [1,2]. Die therapieresistente arterielle
Hypertonie ist definiert als eine nicht leitliniengerechte Blutdruckeinstellung (>140/90 mmHg allgemein) trotz der kontinuierlichen Einnahme einer AntihypertonikaDreifachtherapie unter Einbeziehung eines Diuretikums in geeigneter Kombination [2]. Grundsätzlich sind neben einer medikamentösen Therapie lebensstilmodifizierende Maßnahmen die Basis einer jeden antihypertensiven Behandlung (Abb.3). In der Pathophysiologie der Erkrankung kommt der Überaktivität des vegetativen Nervensystems mit einer Dysbalance aus sympathischer und parasympathischer Aktivität eine besondere Bedeutung zu [21]. Eine Zunahme der efferenten sympathischen Aktivität in der Niere führt zu einer vermehrten Reninausschüttung, vermehrter Natriumretention (proximaler Tubulus) und verminderter renaler Perfusion.

\section{Spironolacton - PATHWAY-2-Studie}

Der blutdrucksenkende Effekt von Aldosteronantagonisten bei Patienten mit therapieresistenter Hypertonie wurde in den letzten Jahren in 2 randomisierten, doppelblinden, placebokontrollierten Studien dokumentiert $[22,23]$. PATHWAY-2 ist die erste Studie, bei der Spironolacton mit anderen Antihypertonika im direkten Vergleich auf seine Wirksamkeit und Verträglichkeit untersucht wurde. Das primäre Ziel der PATHWAY2-Studie war es festzustellen, welche Substanzklasse bei therapieresistentem Bluthochdruck als Therapie der vierten Reihe am besten zum Einsatz kommen könnte [24]. Das sekundäre Ziel war es zu untersuchen, inwieweit die Plasmareninkonzentration einen potenziellen Therapieeffekt vorhersagen kann und ob Spironolacton die effektivste Therapieoption für Patienten mit einer niedrigen Plasmareninkonzentration darstellt: 


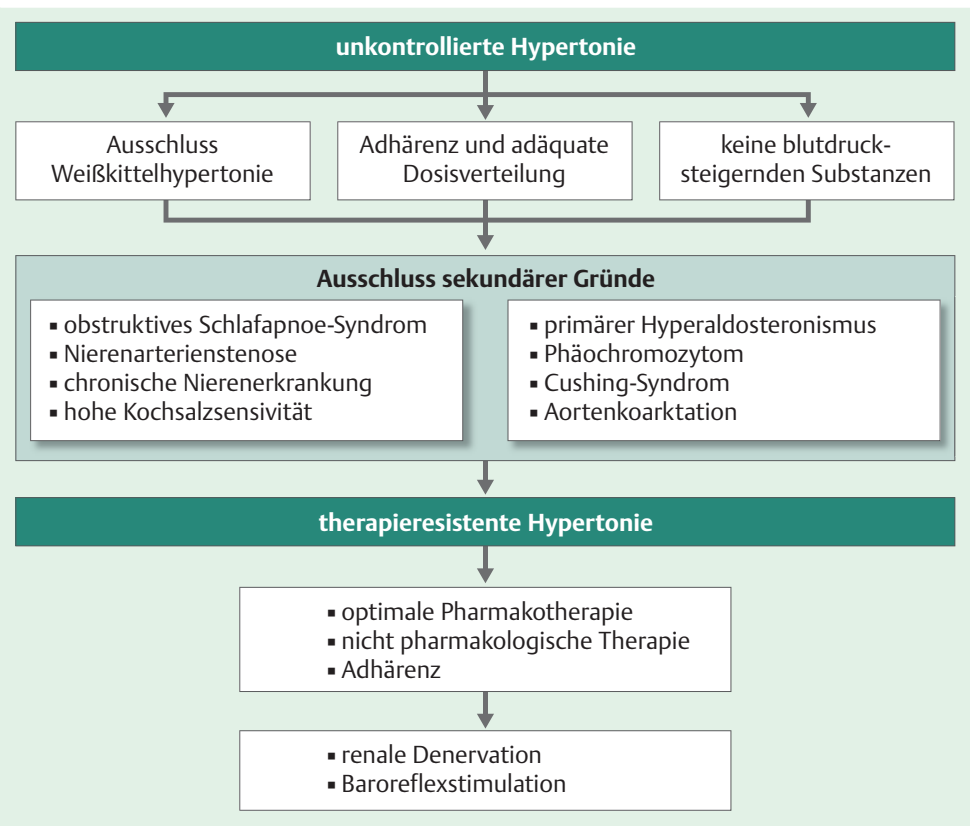

Abb. 3 Systematische Behandlung von Patienten mit resistenter Hypertonie (nach $[2,25])$.
Die Studie weist durch die inverse Beziehung der Plasmareninkonzentration und die durch Spironolacton verursachte Blutdrucksenkung auf die essenzielle Rolle der Natriumretention bei therapieresistenter Hypertonie hin [24].

Spironolacton ist eine effektive Therapieoption bei Patienten mit therapieresistenter Hypertonie. Aufgrund der bekannten Nebenwirkungen in der Langzeittherapie ist jedoch eine regelmäßige Kontrolle der Elektrolyte und renalen Retentionsparameter obligat.

\section{Renale Denervation}

\section{- Verfahren}

Seit geraumer Zeit besteht die Möglichkeit, die renalen Sympathikusfasern durch ein minimal-invasives katheterbasiertes Verfahren gezielt zu veröden [25]. Dazu wird über einen femoralen oder radialen Zugang unter Röntgendurchleuchtung ein Ablationskatheter in die Nierenarterien eingebracht, über den nachfolgend Ablationsenergie abgegeben werden kann. Der Eingriff dauert je nach Device etwa 30-45 Minuten und ist als risikoarm einzustufen, was sowohl durch die Daten der Symplicity HTN-3 [26] als auch des Global Symplicity Registry [27] bestätigt wurde.

\section{Global Symplicity Registry}

In der bislang größten Real-World-Datenbank, dem Global Symplicity Registry [27], zeigte sich bei 1000 Patienten 6 Monate nach der Prozedur eine durchschnittliche Abnahme des systolischen Praxisblutdrucks von $12 \mathrm{mmHg}$ [27]. Bei Patienten mit einem Praxisblutdruck $>160 \mathrm{mmHg}$ bei Studieneinschluss wurde der systolische Blutdruck um $21 \mathrm{mmHg}$ reduziert [27].

\section{- Symplicity HTN-3}

Die Symplicity HTN-3 [26] wurde als US-amerikanische Zulassungsstudie initiiert und schloss erstmals eine Scheinbehandlung ein.

Ergebnisse. Nach 6 Monaten zeigte sich eine Reduktion des systolischen Praxisblutdrucks von $14 \mathrm{mmHg}$ bei den Patienten, die renal denerviert worden waren (RDN-Gruppe), und eine Abnahme von $12 \mathrm{mmHg}$ in der Scheinbehandlungsgruppe. Die Blutdruckunterschiede im Vergleich zum Ausgangsblutdruck waren jeweils signifikant, die Unterschiede zwischen den Gruppen nicht. Für den ambulanten Langzeitblutdruck zeigten sich ähnliche Ergebnisse. 
Diskussionspunkte. Trotz des gut konzipierten Studiendesigns gibt es eine Reihe an Diskussionspunkten, die mittlerweile auch von den Autoren selbst eingeräumt wurden:

- So wurden 364 Patienten der RDN-Gruppe von 111 Untersuchern behandelt, die nur wenig bis keine Erfahrung mit der Prozedur hatten. Weiterführende Analysen deuten darauf hin, dass die technische Qualität der Interventionen einen relevanten Einfluss auf die Blutdruckreduktion hatte [28].

- Die Medikation wurde - trotz der Empfehlung im Studienprotokoll, die blutdrucksenkende Medikation in den ersten 6 Monaten konstant zu halten - bei 38\% der Patienten in der RDN-Gruppe und bei $40 \%$ der Patienten in der Scheinbehandlungsgruppe geändert.

- Des Weiteren wird ein Unterschied in den untersuchten Patientengruppen im Vergleich zu den in Europa behandelten Patienten diskutiert: In der prädefinierten Subgruppe der Kaukasier (Nicht-Afroamerikaner) wurde der primäre Endpunkt einer signifikanten Blutdrucksenkung nach RDN erreicht ( -15 vs. $-9 \mathrm{mmHg}, \mathrm{p}=0,012$ ). Bei den Afroamerikanern zeigte sich dagegen keine unterschiedliche Blutdruckabnahme nach renaler Denervation $(-16 \mathrm{mmHg})$, jedoch eine besonders starke Blutdruckabnahme in der Scheinbehandlungsgruppe $(-18 \mathrm{mmHg})$, was mit der Medikamentenadhärenz zusammenhängen könnte.

\section{DENER-HTN-Studie}

Die multizentrische, randomisierte, kontrollierte DENER-HTN-Studie [29] konnte nachweisen, dass die renale Denervation in Kombination mit einer optimierten medikamentösen Therapie einer alleinigen optimierten medikamentösen Therapie überlegen war.

\section{PRAGUE-15-Studie}

Die prospektive, randomisierte PRAGUE-15-Studie untersuchte den Effekt einer renalen Denervation gegenüber einer intensiven pharmakologischen Therapie mit Spironolacton [30]. Beide Therapien führten nach 6 Monaten zu signifikanten (im Vergleich zum Untersuchungsbeginn), aber zwischen den Gruppen vergleichbaren Blutdrucksenkungen. Die Patienten in der pharmakologischen Gruppe mussten allerdings mehr Medikamente einnehmen und erlitten mehr schwere unerwünschte Ereignisse, wie beispielsweise eine Verschlechterung der Nierenfunktion, die eine langfristige Therapie mit Spironolacton verkompliziert.

\section{SPYRAL-HTN-Studien}

Um sich Klarheit darüber zu verschaffen, welche Bedeutung die renale Denervation als antihypertensive Therapie hat, wurden in jüngster Zeit eine Reihe neuer Studienprogramme aufgelegt [31]. Die SPYRAL-HTNStudien untersuchen dabei den Effekt einer renalen Denervation bei Patienten mit unkontrolliertem Bluthochdruck. Eingeschlossen werden lediglich Patienten mit einer kombinierten (>140/>90 mmHg) Hypertonie mit einem systolischen Praxisblutdruck von 150 $180 \mathrm{mmHg}$ und einem systolischen 24-Stunden-Blutdruck von $140-170 \mathrm{mmHg}$.

Gruppen. Die SPYRAL-HTN-Studien sind prospektiv, randomisiert und doppelblind angelegt und werden mit einer Scheinbehandlung kontrolliert. Die Patienten gehören einer von 2 Gruppen an:

- In der Antihypertensiva-naiven OFF-MED-Gruppe (NCT02439749) wird bis mindestens 3 Monate nach dem Eingriff auf eine antihypertensive Medikation verzichtet.

- In der ON-MED-Gruppe (NCT02439775) werden Patienten mit therapieresistentem Bluthochdruck mit einer antihypertensiven Dreifachtherapie als Fixkombination (Thiaziddiuretikum, DihydropiridintypKalziumantagonist und ACE-Hemmer oder Angiotensinrezeptorblocker) behandelt (Abb. 4).

Änderungen zur SYMPLICITY-HTN-Studie. Das Studiendesign von SPYRAL-HTN beinhaltet einige relevante Änderungen im Vergleich zu den SYMPLICITY-HTNProtokollen:

- Erstmals wird ein Multielektrodenkatheter eingesetzt, der aufgrund seines prädefinierten Designs eine zirkumferenzielle 4-Quadranten-Ablation ermöglicht.

- Sowohl der Hauptstamm der Nierenarterie, die distalen Segmentarterien der Nieren sowie akzessorische Nierenarterien werden ebenfalls mittels Ablationskatheter behandelt und in die Therapieplanung eingeschlossen.

- Der Eingriff wird an Zentren durchgeführt, die Erfahrung mit der renalen Denervation haben. Pro Zentrum nimmt den Eingriff immer der gleiche Operateur vor.

Primärer Endpunkt der Studien ist die systolische 24Stunden-Blutdruckreduktion nach 3 Monaten. Eine vergleichbare Untersuchung unter Einsatz eines ballonbasierten Ablationskatheters (Vessix Reduce Renal Denervation System [Boston Scientific]) erfolgt in den USA unter dem Namen REDUCE-HTN (NCT02392351). 


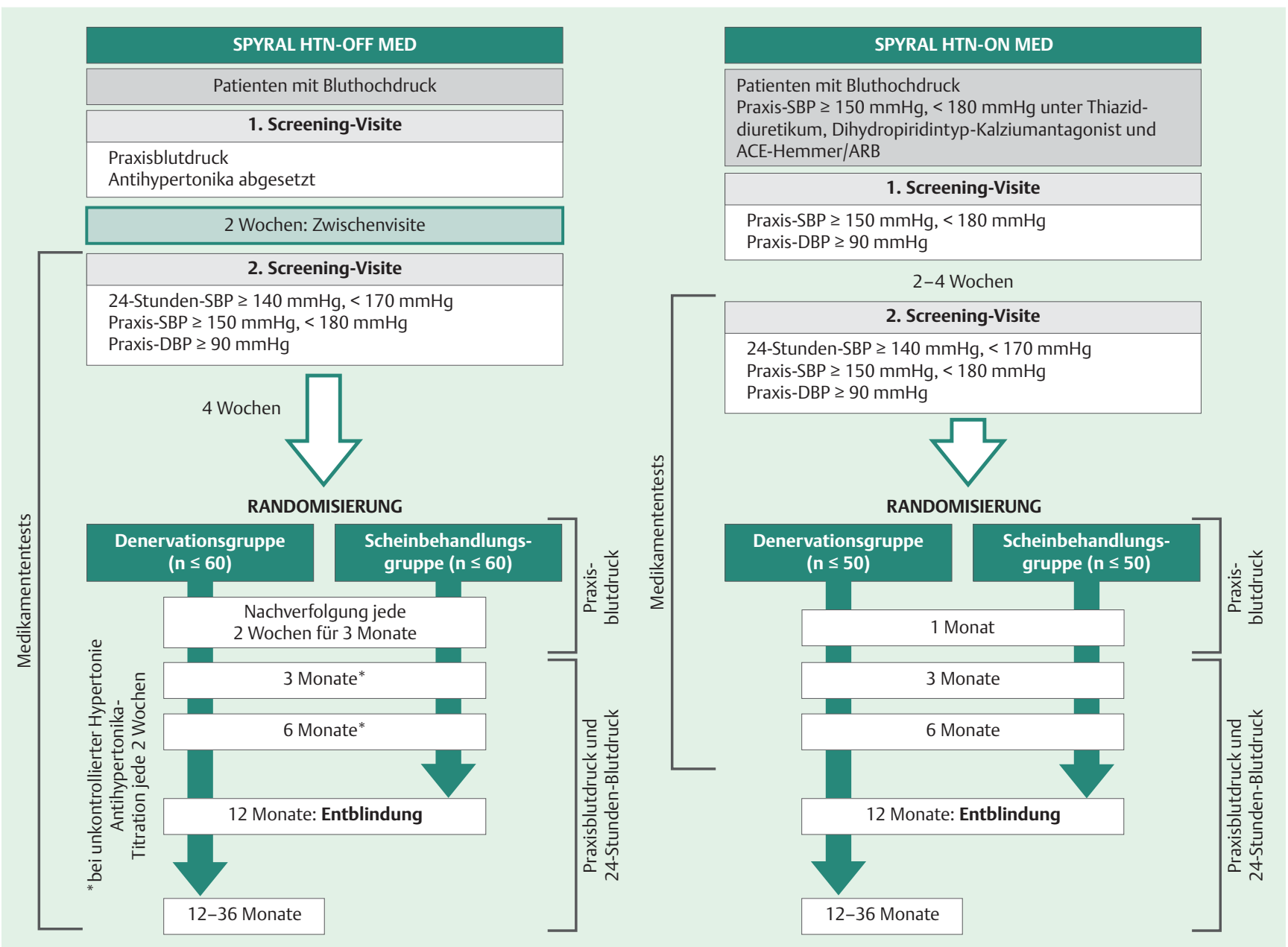

Abb. 4 Design der SPYRAL-HTN-OFF-MED (links) und der SPYRAL-HTN-ON-MED (rechts); ARB = Angiotensinrezeptorblocker, SBP= systolischer Blutdruck, DBP= diastolischer Blutdruck (nach [31]).

Die Ergebnisse der neuen Studien werden wichtige Erkenntnisse liefern.

Die folgenden Fragen beziehen sich auf den vorangehenden Beitrag. Bitte schicken Sie uns die entsprechenden Lösungsbuchstaben. Jeweils eine Antwort ist richtig. Die Vergabe von CME-Punkten ist an die korrekte Beantwortung der Multiple-Choice-Fragen gebunden.
Interessenkonflikt: FM gibt an, dass er Honorare der Firmen Medtronic/Ardian und St. Jude Medical erhalten hat. MB gibt an, dass er Honorare der Firmen Servier, Medtronic und Boehringer Ingelheim erhalten hat. 


\section{Kernaussagen}

- Die aktuelle Leitlinie empfiehlt einen Zielblutdruckwert von $<140 / 90 \mathrm{mmHg}$. Die Ergebnisse der SPRINT-Studie zeigen, dass ein SBP unter $120 \mathrm{mmHg}$ im Vergleich zu einem SBP unter $140 \mathrm{mmHg}$ - die Inzidenz kardiovaskulärer Ereignisse bei Hochrisikopatienten um ungefähr ein Drittel und die Mortalität um ungefähr ein Viertel reduzieren kann.

- Hypokaliämie und Glukoseintoleranz sind Nebenwirkungen unter Therapie mit einem Thiaziddiuretikum. Die PATHWAY-3-Studie ergab, dass eine Kombination von HCT und Amilorid eine effektive Blutdrucksenkung ohne Kaliumund Glukoseveränderungen ermöglicht.
- LCZ696 führt zu einer kombinierten Hemmung des RAS und einer Steigerung natriuretischer Peptide. Hierdurch werden Natriumausscheidung, Vasodilatation und Kardioprotektion vermittelt. In der PARAMETER-Studie war eine 12-wöchige Therapie mit LCZ696 bei Hochrisikopatienten mit isoliert systolischer Hypertonie mit einer effektiven Senkung des zentralen Blutdrucks und Pulsdrucks, des 24-Stunden-Blutdrucks sowie der NT-pro-BNP-Level assoziiert. Diese Abnahme war deutlicher als unter einer Therapie mit einem Angiotensinrezeptorblocker allein.

- Spironolacton ist eine effektive Therapieoption bei Patienten mit therapie-

\section{Abstract}

High blood pressure remains a leading risk factor for cardiovascular death worldwide. Optimal medical therapy and blood pressure control are important for the prevention of cardiovascular and renal disease. The present review aims to provide an overview about the innovations and novelties in the field of hypertension. First, new findings concerning optimal blood pressure goals in high-risk patients are presented. Second, developments of established medication regimes, particularly thiazide diuretic, as well as innovations, the angiotensin receptor-neprilysin inhibitor, are reviewed. Finally, pharmacological and non-pharmacological therapeutic options for resistant hypertension are discussed, such as a recent study investigating spironolactone as a 4th-line agent and renal denervation as a continuously evolving treatment option.

\section{Über die Autoren}

\section{Ina Zivanovic}

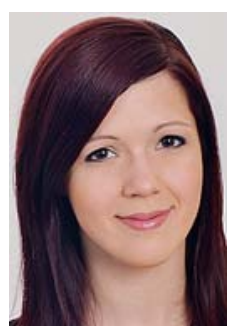

Dr. med. 2006 - 2014 Studium der Humanmedizin an der Universität Ljubljana, Slowenien. Seit 2015 Forschung an der Klinik für Innere Medizin III/Kardiologie, Angiologie und Internistische Intensivmedizin am Universitätsklinikum des Saarlandes in Homburg/Saar.

\section{Sebastian Ewen}

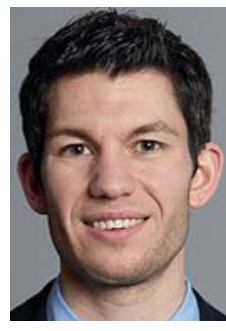

Dr. med. 2005-2011 Studium der Humanmedizin an der Philipps-Universität Marburg. Seit 2012 Assistenzarzt an der Klinik für Innere Medizin III, Kardiologie, Angiologie und Internistische Intensivmedizin am Universitätsklinikum des Saarlandes in Homburg/Saar.

\section{Michael Böhm}

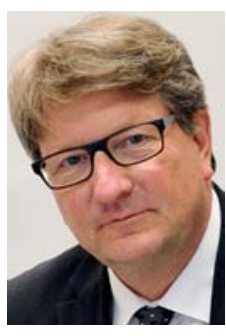

Prof. Dr. med. 1977 - 1984 Medizinstudium an der Medizinischen Hochschule Hannover. 1984-1986 Assistent im Institut für Allgemeine Pharmakologie des Universitätsklinikums Hamburg Eppendorf. 1986-1993

Assistenzarzt an der Klinik für Innere Medizin I, Kardiologie des Klinikums Großhaderns, Ludwig-Maximilians-Universität München. 1993-2000 Oberarzt an der Klinik für Innere Medizin III, Kardiologie an der Universität Köln. Seit 2000 Direktor der Klinik für Innere Medizin III, Kardiologie, Angiologie und Internistische Intensivmedizin am Universitätsklinikum des Saarlandes in Homburg/Saar. 


\section{Felix Mahfoud}

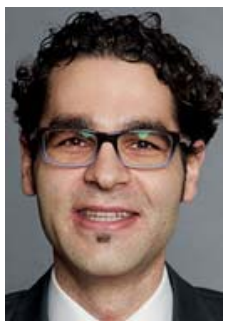

Priv.-Doz. Dr. med. 2001 - 2007 Studium der Humanmedizin an der Johann Wolfgang Goethe-Universität, Frankfurt am Main. 2007-2014 Wissenschaftlicher Assistent, seit 2015 Oberarzt an der Klinik für Innere Medizin III, Kardiologie, Angiologie und Internistische Intensivmedizin am Universitätsklinikum des Saarlandes in Homburg/Saar.

\section{Korrespondenzadresse}

Priv.-Doz. Dr. med. Felix Mahfoud

Klinik für Innere Medizin III

Kardiologie, Angiologie und Internistische

Intensivmedizin

Universitätsklinikum des Saarlandes

Kirrberger Straße

66421 Homburg/Saar

E-Mail: Felix.Mahfoud@uniklinikum-saarland.de

\section{Literatur}

1 Lim SS, Vos T, Flaxman AD et al. A comparative risk assessment of burden of disease and injury attributable to 67 risk factors and risk factor clusters in 21 regions, 1990-2010: a systematic analysis for the Global Burden of Disease Study 2010. Lancet 2012; 380: 2224-2260

2 Mancia G, Fagard R, Narkiewicz K et al. 2013 ESH/ESC guidelines for the management of arterial hypertension: the Task Force for the Management of Arterial Hypertension of the European Society of Hypertension (ESH) and of the European Society of Cardiology (ESC). Eur Heart J 2013; 34: 2159-2219

3 Ambrosius WT, Sink KM, Foy CG et al. The design and rationale of a multicenter clinical trial comparing two strategies for control of systolic blood pressure: the Systolic Blood Pressure Intervention Trial (SPRINT). Clin Trials 2014; 11: 532-546

4 Wright JT, Williamson JD, Whelton PK et al. A randomized trial of intensive versus standard blood-pressure control. N Engl ] Med 2015: [in press]

5 Sundstrom J, Arima H, Woodward M et al. Blood pressurelowering treatment based on cardiovascular risk: a meta-analysis of individual patient data. Lancet 2014; 384: 591 - 598

6 Cushman WC, Evans GW, Byington RP et al. Effects of intensive blood-pressure control in type 2 diabetes mellitus. N Engl J Med 2010; 362: 1575-1585

7 Lee M, Saver JL, Hong KS et al. Does achieving an intensive versus usual blood pressure level prevent stroke? Ann Neurol 2012; 71: $133-140$

8 Benavente OR, Coffey CS, Conwit R et al. Blood-pressure targets in patients with recent lacunar stroke: the SPS3 randomised trial. Lancet 2013; 382: 507-515
9 Brown MJ, Williams B, MacDonald TM et al. Comparison of single and combination diuretics on glucose tolerance (PATHWAY-3) protocol for a randomized double-blind trial in patients with essential hypertension. BM] Open 2015; 5: e008086

10 Brown MJ, Williams B, Morant SV et al. Effect of amiloride, or amiloride plus hydrochlorothiazide, versus hydrochlorothiazide on glucose tolerance and blood pressure (PATHWAY-3): a parallel group double-blind randomized phase 4 trial. Lancet Diabetes Endocrinol 2015: [Epub ahead of print]

11 Ogawa $\mathrm{H}$, Soejima H, Matsui K et al. A trial of telmisartan prevention of cardiovascular diseases (ATTEMPT-CVD): Biomarker study. Eur J Prev Cardiol 2015: [Epub ahead of print]

12 Packer M. Evolution of the neurohormonal hypothesis to explain the progression of chronic heart failure. Eur Heart J 1995; 16: 4-6

13 Ruilope LM, Dukat A, Böhm M et al. Blood-pressure reduction with LCZ696, a novel dual-acting inhibitor of the angiotensin II receptor and neprilysin: a randomised, double-blind, placebo-controlled, active comparator study. Lancet 2010; 375: $1255-1266$

14 Bevan EG, Connell JM, Doyle J et al. Candoxatril, a neutral endopeptidase inhibitor: efficacy and tolerability in essential hypertension. J Hypertens 1992; 10: 607-613

15 Packer M, Califf RM, Konstam MA et al. Comparison of omapatrilat and enalapril in patients with chronic heart failure: the Omapatrilat Versus Enalapril Randomized Trial of Utility in Reducing Events (OVERTURE). Circulation 2002; 106: 920 926

16 Kostis JB, Packer M, Black HR et al. Omapatrilat and enalapril in patients with hypertension: the Omapatrilat Cardiovascular Treatment vs. Enalapril (OCTAVE) trial. Am J Hypertens 2004; 17: $103-111$

17 McMurray JI, Packer M, Desai AS et al. Angiotensin-neprilysin inhibition versus enalapril in heart failure. N Engl J Med 2014; 371: $993-1004$

18 Jhund PS, Claggett B, Packer M et al. Independence of the blood pressure lowering effect and efficacy of the angiotensin receptor neprilysin inhibitor, LCZ696, in patients with heart failure with preserved ejection fraction: an analysis of the PARAMOUNT trial. Eur J Heart Fail 2014; 16: 671 -677

19 Williams B, Cockcroft JR, Kario K et al. Rationale and study design of the Prospective comparison of Angiotensin Receptor neprilysin inhibitor with Angiotensin receptor blocker MEasuring arterial sTiffness in the eldERly (PARAMETER) study. BMJ Open 2014; 4: e004254

20 Williams B, Cockcroft JR, Kario K et al. Principal results of the Prospective comparison of Angiotensin Receptor neprilysin inhibitor with Angiotensin Receptor blocker MEasuring arterial sTiffness in the eldERly (PARAMETER) Study. London: ESC Congress 2015; oral presentation 31. 082015

21 DiBona GF. Physiology in perspective: The Wisdom of the Body. Neural control of the kidney. Am J Physiol Regul Integr Comp Physiol 2005; 289: R633-641

22 Vaclavik J, Sedlak R, Plachy M et al. Addition of spironolactone in patients with resistant arterial hypertension (ASPIRANT): a randomized, double-blind, placebo-controlled trial. Hypertension 2011; 57: 1069-1075

23 Oxlund CS, Henriksen JE, Tarnow L et al. Low dose spironolactone reduces blood pressure in patients with resistant 
hypertension and type 2 diabetes mellitus: a double blind randomized clinical trial. J Hypertens 2013; 31: 2094-2102

24 Williams B, MacDonald TM, Caulfield M et al. Prevention And Treatment of Hypertension With Algorithm-based therapy (PATHWAY) number 2: protocol for a randomised crossover trial to determine optimal treatment for drug-resistant hypertension. BMJ Open 2015; 5: e008951

25 Ewen S, Ukena C, Böhm M et al. Percutaneous renal denervation: new treatment option for resistant hypertension and more? Heart 2013; 99: 1129-1134

26 Bhatt DL, Kandzari DE, O'Neill WW et al. A controlled trial of renal denervation for resistant hypertension. $N$ Engl J Med 2014; 370: 1393-1401

27 Böhm M, Mahfoud F, Ukena C et al. First report of the Global SYMPLICITY Registry on the effect of renal artery denervation in patients with uncontrolled hypertension. Hypertension 2015; 65: 766-774

28 Kandzari DE, Bhatt DL, Brar S et al. Predictors of blood pressure response in the SYMPLICITY HTN-3 trial. Eur Heart J 2015; 36: $219-227$
29 Azizi M, Sapoval M, Gosse P et al. Optimum and stepped care standardised antihypertensive treatment with or without renal denervation for resistant hypertension (DENERHTN): a multicentre, open-label, randomised controlled trial. Lancet 2015; 385: $1957-1965$

30 Rosa J, Widimsky P, Tousek P et al. Randomized comparison of renal denervation versus intensified pharmacotherapy including spironolactone in true-resistant hypertension: six-month results from the Prague-15 study. Hypertension 2015; 65: 407-413

31 Kandzari DE, Kario K, Mahfoud F et al. The SPYRAL HTN Global Clinical Trial Program: Rationale and design for studies of renal denervation in the absence (SPYRAL HTN OFF-MED) and presence (SPYRAL HTN ON-MED) of antihypertensive medications. Am Heart ] 2015: [in press] 


\section{CME.thieme.de}

\section{CME-Fragen}

\section{CME-Teilnahme}

- Viel Erfolg bei lhrer CME-Teilnahme unter http://cme.thieme.de

- Diese Fortbildungseinheit ist 12 Monate online für eine CME-Teilnahme verfügbar.

- Sollten Sie Fragen zur Online-Teilnahme haben, unter http://cme.thieme.de/hilfe finden Sie eine ausführliche Anleitung.

1 Welche der folgenden Aussagen ist richtig? In der SPRINT-Studie führte ein SBP-Zielwert unter $120 \mathrm{mmHg}$ verglichen mit einem Zieldruck unter $140 \mathrm{mmHg}$ zu einer Reduktion von kardiovaskulären Ereignissen und Mortalität bei Patienten mit Bluthochdruck und ...?
A Diabetes mellitus
B Zustand nach Schlaganfall
C einer glomerulären Filtrationsrate (GFR) $<20 \%$
D Herzinsuffizienz mit Pumpfunktion $<35 \%$
E 10-Jahres-Framingham-Risiko-Score $\geq 15 \%$

2 Wie hoch ist den Leitlinien nach der empfohlene ambulante 24-Stunden-Zielblutdruck?
A $\leq 120 / 80 \mathrm{mmHg}$
B $\leq 140 / 90 \mathrm{mmHg}$
C $\leq 135 / 85 \mathrm{mmHg}$
D $\leq 130 / 80 \mathrm{mmHg}$
E $\leq 120 / 70 \mathrm{mmHg}$

3 Welche der folgenden Substanzen wurde in der PATHWAY-3-Studie mit HCT kombiniert, um die Entstehung einer Glukoseintoleranz zu verhindern?
A Amilorid
B Triamteren
C Spironolacton
D Eplerenon
E Ramipril

4 Welche der folgenden Aussagen ist richtig? Die ATTEMPT-CVD-Studie verzeichnete bei einer Telmisartan-Therapie verglichen mit einer Standard-Bluthochdrucktherapie ...
A eine Erhöhung des Plasma-BNP
B eine Erhöhung des Urin-Albumin/Kreatinin-Quotienten
C eine erhöhte Inzidenz kardiovaskulärer Ereignisse
D eine Senkung des Urin-Albumin/Kreatinin-Quotienten
E eine niedrigere Inzidenz kardiovaskulärer Ereignisse

5 Welche der folgenden Aussagen ist richtig? Die Substanz LCZ696 ist ...
A ein Neprilysininhibitor
B ein Hybridmolekül aus Diuretikum und Neprilysininhibitor
C ein Hybridmolekül aus Angiotensinrezeptorblocker und Neprilysininhibitor
D ein Neprilysinagonist
E ein Hybridmolekül aus ACE-Hemmer und Neprilysininhibitor 


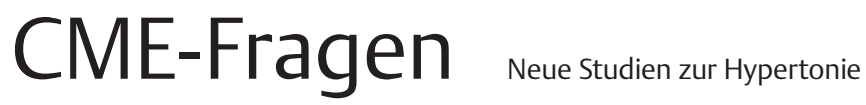

6 Welche der folgenden Aussagen ist richtig? Die Bluthochdrucktherapie mit LCZ696 führte in der PARAMETER-Studie vor allem zur Senkung des/der ...
A Blutdrucks im nächtlichen Intervall
B Blutdrucks im Tagesintervall
C 24-Stunden-Blutdrucks
D Praxisblutdrucks
E Blutdruckvariabilität

7 Welche der folgenden Substanzen war in der PATHWAY-2-Studie die erfolgreichste Therapie der vierten Reihe bei Patienten mit einer therapieresistenten Hypertonie?
A Bisoprolol
B Doxazosin
C Moxonidin
D Spironolacton
E Amilorid

8 Was ist die Folge einer Zunahme der efferenten sympathischen Aktivität in der Niere?
A eine verminderten Reninausschüttung
B eine vermehrte Natriumretention (proximaler Tubulus)
C eine vermehrte renale Perfusion
D eine verminderte Wasserresorption
E eine verminderte Natriumretention (proximaler Tubulus)

9 Wie ist die Definition einer therapieresistenten arteriellen Hypertonie?

A Blutdruckwerte $>130 / 85 \mathrm{mmHg}$ trotz der Einnahme eines Diuretikums

B Blutdruckwerte $>140 / 90 \mathrm{mmHg}$ trotz der kontinuierlichen Einnahme einer antihypertensiven Dreifachtherapie unter Einbeziehung eines Diuretikums

C Blutdruckwerte $>160 / 90 \mathrm{mmHg}$ und linksventrikuläre Hypertrophie

D Blutdruckwerte $>130 / 85 \mathrm{mmHg}$ trotz der Einnahme eines ACE-Hemmers, Angiotensinrezeptorblockers, Kalziumantagonisten und Betablockers

E Blutdruckwerte $>140 / 90 \mathrm{mmHg}$ trotz der kontinuierlichen Einnahme eines Angiotensinrezeptorblockers, Kalziumantagonisten und Betablockers

10 Was gehört nicht zur Routinediagnostik vor einer renalen Denervation?
A Doppler/Duplex-Ultraschall zum Ausschluss einer Nierenarterienstenose
B Aldosteron-Renin-Ratio zum Ausschluss eines Conn-Syndroms
C 24-Stunden-Langzeitblutdruckmessung zum Ausschluss eines situativ erhöhten Bluthochdrucks
D Kontrolle der Metaboliten antihypertensiver Substanzen im Urin zur Kontrolle der Medikamenten-Compliance
E renale Retentionsparameter zur Bestimmung der Nierenfunktion 